\title{
ACTIVATION OF PRO-APOPTOTIC CELLS, REACTIVE ASTROGLIOSIS AND HYPERPHOSPHORYLATION OF TAU PROTEIN IN TRIMETHYLTIN-INDUCED HIPPOCAMPAL INJURY IN RATS.
}

\begin{abstract}
1Okesina AA, 2Ajao MS, 1Buhari MO, 1Afodun AM, 3Okesina KB, 2Usman RY, 2Sulaimon FA, 1Kolawole BP
1Department of Human Anatomy, Faculty of Biomedical Sciences Kampala International University, Uganda.
\end{abstract}

2Department of Anatomy, Faculty of Basic Medical Sciences, College of Health sciences, University of Ilorin, Ilorin, Nigeria.

з Optometry Department, Faculty of Life Sciences, University of Ilorin, Ilorin, Nigeria.

Correspondence to Ayodeji A. Okesina, PhD, Department of Human Anatomy, Faculty of Biomedical Sciences, Kampala International University, P.O. BOX 71, Busenyi, Uganda.

akeemokesina@gmail.com, +256757847741

\begin{abstract}
Neurodegenerative diseases cause neural cells to lose both the functional and sensory abilities as a result of genetic factors, proteopathies and mitochondrial dysfunction. Neurodegeneration forms the basis of most neurodegenerative disorders for example Alzheimer's disease, Huntington's diseases, and Parkinson's diseases. The mechanism that underlines the process of neurodegeneration is not well understood. Understanding the process and mechanism involved in neurodegeneration might offer a better therapeutic approach to positively manage cases of neurodegenerative diseases. Therefore, this study's target was to create an animal model to study neurodegeneration. Sixteen adult male Wistar rats were used in the study and divided into two groups. Control (0.2 mL of normal saline (NS)), and trimethyltin-treated (TMT, $8 \mathrm{mg} / \mathrm{kg}$ stat dose only). These animals underwent perfusion with $4 \%$ paraformaldehyde, brain excision and analysis of p53 antigen, GFAP and Bielshowsky on these tissues. The results showed that animals in the control group showed presence of activated p53 antigen, reactive astrogliosis, neurofibrillary tangles, and amyloid plaques within the cytoplasm of the hippocampal cells. Cornus Ammonis (CA2) and (CA3) showed more of the trimethylrtin injury than CA1 and CA4. This study thus revealed that, intra-peritoneal administration of single dose of $8 \mathrm{mg} / \mathrm{kg}$ of trimethyltin can offer an attractive disease model to study some neurodegenerative diseases.
\end{abstract}

Keywords: p53 antigen, Bielshowsky, Glia fibrillary acidic protein, Trimethyltin, Hippocampus,

\section{INTRODUCTION}

Neurodegeneration is a gradual process that leads to the loss of structures and functions of a neuron (i.e. neuronal death). Neurodegeneration forms the basis of most neurodegenerative disorders such as Alzheimer's disease,
Huntington's diseases, and Parkinson's diseases. (Rubinsztein, 2006). Neurodegenerative diseases share a common feature, which includes accumulation of disease-specific proteins into insoluble aggregates that causes 
neuronal dysfunction. The neurodegenerative disorders affect millions of people worldwide, because of its progressive nature which poses a lot of problems to the field of medicine (Price et al., 1998).

Trimethyltin (TMT) induces its neurodegeneration ability in several ways, but it particularly characterised by massive neuronal death, mainly localised in the limbic system, especially in the hippocampus. the cell death accompanied by several conditions like reactive gliosis, epilepsy and marked neurobehavioral alterations (Geloso et al., 2004). This is why TMT-intoxicated rats an attractive model to study neurodegenerative diseases such as Alzheimer's disease (Woodruff et al., 1994). Understanding the pathophysiology i.e. components of cell affected might be essential in developing disease-modifying strategies able to halt their progression. (Colpo and Teixeira, 2017) and to formulate drugs that will manage and treat several types neurodegenerative diseases.

\section{MATERIALS AND METHODS}

Sixteen adult male rats (Rattus novergicus), weighing between $220-250 \mathrm{~g}$ were used for this study. The rats were procured from the animal holdings in the Department of Zoology, University of Ilorin, and were allowed to acclimatize in the animal house of the College of Health Sciences, University of Ilorin for 14 days prior to commencement of the experiments. The animals were housed in cages under normal light/dark cycle and at room temperature/humidity. Food and water were available ad libitum. Ethical approval for this study was obtained from the University of Ilorin Ethical Review Committee. The rats were randomly divided into two groups (Control and TMT, $\mathrm{n}=8$ per group). The animals in the control group were given $0.2 \mathrm{~mL}$ of normal saline (vehicle, i.p.) for 21 days. TMT was given trimethyltin ( $8 \mathrm{mg} / \mathrm{kg}$, i.p.) only on the first day, and were monitored for 21 days (Brock and O'Callaghan 1987). TMT rats were sacrificed on day 21.

The open end of perfusion tube was placed in a beaker filled with cold $4 \%$ paraformaldehyde (in ice box). The giving sets were adjusted to a slow steady drip of about ( $20 \mathrm{ml} / \mathrm{min})$. An appropriate amount of anaesthetic agent (Ketamine 20 $\mathrm{mg} / \mathrm{kg}$ ) was given intra-muscularly to the animals. When it was noticed that the animal was under the influence of the anaesthesia, it was placed on the operating table with its back down. The appendages of the animal were then pinned down to a board on the operating surface. Pinch response method was done to determine depth of anaesthesia and the animal was unresponsive. Incision was made by a scalpel through the abdomen to the length of the diaphragm. A sharp scissor was then used to cut through the connective tissue at the bottom of diaphragm to allow access to rib cage. After which a large scissor, blunt side down, was used to cut through ribs just left of the rib cage midline. Two end centres horizontal cuts were done through the rib cage, to open up thoracic cavity. A Clamp was used to expose heart and provide drainage for blood and fluids. Forceps was then used to hold the heart steady while it was still beating. A needle was inserted into the protrusion of the left ventricle which extends to about $5 \mathrm{~mm}$. I was careful not go deeper so as not to damage any interior wall and compromise circulation. The needle position was secured by clamping just near the point of entry. The valve was released to allow slow, steady flow of around $20 \mathrm{ml} / \mathrm{min}$ of $0.9 \%$ saline solution. A cut was made in the atrium with sharp scissors to ensure solution flows freely. This lasted for about 8-7 minutes, after which the solution was changed to $4 \%$ paraformaldehyde. Perfusion was confirmed by spontaneous movement (formalin dance) and lightened colour of the liver was observed. Perfusion was stopped and brain was removed and fixed in the same fixative for 
another 2 hours at 4oC overnight before starting dehydration and embedding, modified from Ajao et al., (2010).

Tissue Collection After perfusion has been completed, the whole brain tissues were excised and were post-fixed in $4 \%$ paraformaldehyde overnight. The whole hippocampal CA regions were excised and equilibrated in $30 \%$ sucrose solution, before histochemical and immunohistochemical analysis. The sections were taken at $2 \mu \mathrm{m}$ on paraffin wax embedded tissue blocks and mounted on a glass slide. All antibodies were procured from Dianova (GmbH/Warbugstr. 45/20354 Hamburg. Also, reagents and buffers used in this study were molecular biology grade ( $99.9 \%$ pure) from Sigma-Aldrich. Bieshowsky Stain was used to visualise nerve fibre, neurofibrillary tangles and amyloid plaques in hippocampal cells death.

P53 antigen acts as a tumor suppressor in many tumor types. It was used to indicate proapoptotic cells during cell cycle in the hippocampus of adult male Wistar rats. The method employed was the avidin biotin complex method also referred to the avidin biotin immunoperoxidase method. The antibody dilution factor used was 1:100 for all the antibody markers. The processed tissues were sectioned at two microns on the rotary microtome and placed on a hotplate at $70{ }^{\circ} \mathrm{C}$ for at least an hour.

The images were acquired using an Optronics Digital Camera connected to a computer interface (MagnaFire) and an Olympus BX-51 research microscope. The total cell count was determined using a $10 \times 10$ grid graticle at $400 \times$ magnification. For the corona sections, $\mathrm{n}=2$ fields were captured to obtain $n=4$ fields per section. Subsequently, the average count for $n$ $=4$ fields was determined as the representative value (Ogundele et al. 2015). Immunopositive cells in P53 was counted using software package called image J (1.48v). The statistical package for social sciences (SPSS, version 20) was used for statistical analysis and Graphpad Prism (version 5.01) was used to draw the bar charts. All data were analysed using one-way analysis of variance. All values were reported as Mean \pm standard error of mean (SEM) and $p<0.05$ was considered statistically significant.

\section{RESULTS}

Activation of p53 antigen: The administration of normal saline (NS) produced few amounts of p53 antibodies (Figure 1A) in the hippocampus of the rat. There were more visible amounts of p53 antibodies (Figure 1B). Also, there exist significant increase in the level of p53 antibodies in the hippocampus of rats treated with trimethyltin (TMT) when compared to rats that received normal saline (NS), which indicated the presence of pro-apoptotic cells in the hippocampus (Figure 1).

The process of identifying reactive astrogliosis in trimethyltin treated hippocampus of rat. There were observable astrocytes connections in the hippocampus of rats that were given normal saline (Figure 2A). The hippocampus of rats that received trimethyltin as shown (Figure 2B), pictured high level of abnormal astrocytes proliferation which indicated astrogliosis and in some extreme cases led to the formation of scar.

Histochemical analysis of the effect of trimethyltin on the hippocampus: The rats that received normal saline (NS) shows normal histoarchitectural appearance (Figure 3A); presence of normal structural proteins. This was not the same for the rats that were treated with trimethyltin (TMT) because there was substantial tissue damage (Figure 3B), which was evident, as a result of visible neurofibrillary tangles and plaque formation around the cells of the hippocampus. 


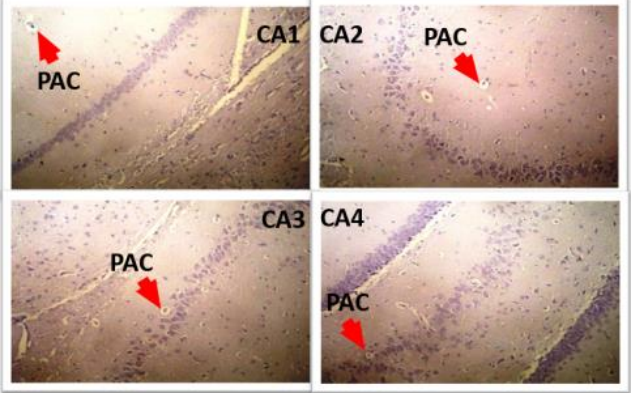

Fig. $1 \mathrm{~A}$

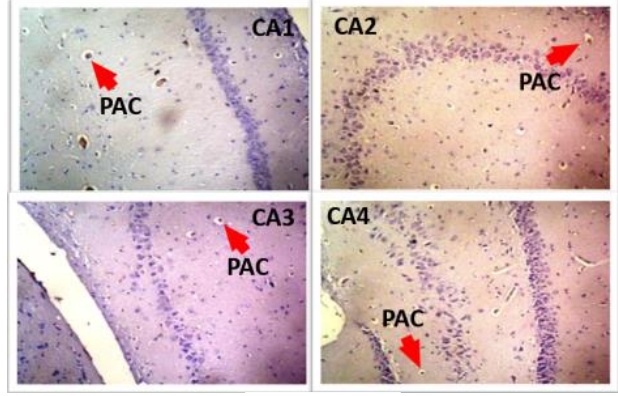

Fig. 1B

Figure 1(1A-B): Representative photomicrographs with p53 antibody on Cornus Ammonis (CA) $1,2,3$ and 4 of the hippocampus of adult male Wistar rats. Red arrows points to activated p53 antigens; pro-apoptotic cells (PAC) in the hippocampus

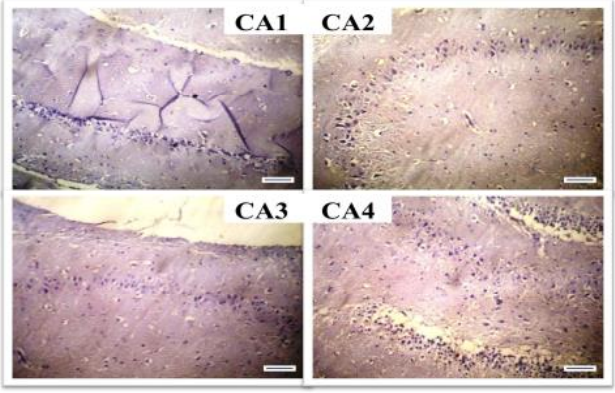

Fig. $2 \mathrm{~A}$

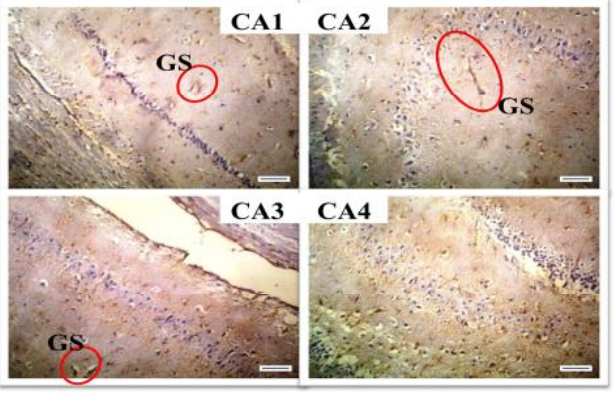

Fig. 2B

Figure 2: Representative photomicrographs of immunohistochemical (Glial fibrillary acidic protein GFAP) staining of the Cornus Ammonis (CA) 1, 2, 3 and 4. Fig 2A; Array of normal astrocytes connections found within the hippocampus. Fig 2B; Details of glia scar (GS) were observed as indicated in the red rings in the hippocampus.

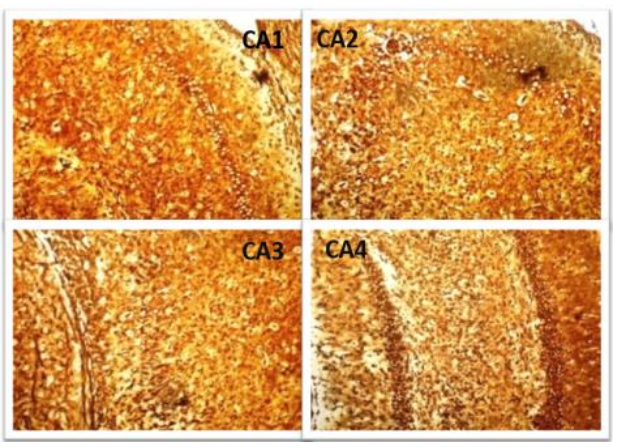

Fig. 3A

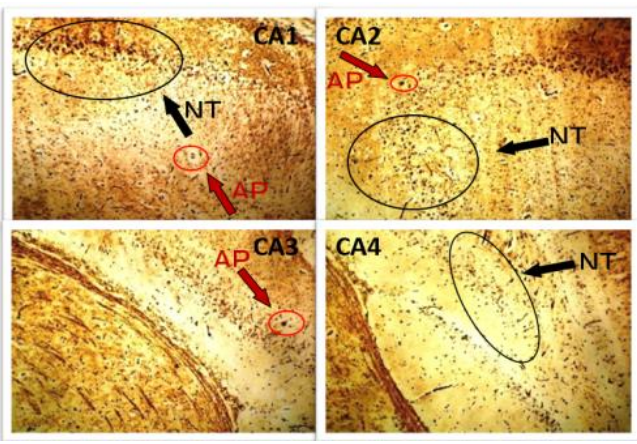

Fig. 3B

Figure 3: Representative photomicrographs of histochemical (Bielshowsky) staining of the Cornus Ammonis (CA) 1, 2, 3 and 4. Fig 3A; components of normal cellular protein filaments. Fig 3B; Details of amyloid plaques (AP) indicated by red arrows and neurofibrillary tangles (NT) indicated by black arrows, found within the cells of the hippocampus. 
Anatomy Journal of Africa. 2020. Vol 9 (1): 1782 - 1788.

\section{DISCUSSION}

Neurodegeneration is the gradual deterioration in a person's cognitive abilities, such as memory. This deterioration may be due to some structural changes that prevent the neurons from functioning normally (Bredesen et al., 2006). For instance, this current study focused on mimicking an animal diseased model created by using intraperitoneal administration of trimethyltin on rats to achieve hippocampal injury and histochemical and immunohistochemical analysis were done to confirm the progression of these injuries. TMT has been found useful as experimental model in the study of Alzheimer-like diseases (Nilsberth et al., 2002). The pathological features that have been achieved by TMT induced neurodegeneration is common to most neurodegenerative disorders, like neuronal cell death and cognitive impairment (Nilsberth et al., 2002).

The administration of normal saline in this study revealed that, there was no evidence of protein deformation, abnormal proliferation of astrocytes and cellular death in the cornus ammonis (CA) CA1 to CA4 of the hippocampus of rats. But the rats that were treated with trimethyltin showed series abnormal events. For example, the rats that received single dose of trimethyltin alone showed diffused neurofibrillary tangles plaque formation in the regions of the hippocampus. This finding suggested exposure of the rats to trimethyltin caused hyper-phosphorylation of tau proteins in the hippocampus, allowing them to dissociate themselves from adjoining microtubules. The tau protein clusters with each other to form tangles in the hippocampal regions of these rats. This result is supported by the work of Constantinidis, (1991) and Yokel, (2000), that reported neurotoxins like trimethyltin and aluminium respectively contributes to formation of neurofibrillary tangles and plaques when exposed to the brain.

In addition, to identify the pattern of reactive astrogliosis after treatment with trimethyltin,
GFAP was used to show the expression of astrocytes. The rats received single dose of trimethyltin only showed multiple expressions of astrocytes in the regions of the hippocampus, which also led to scar formation in some cases. astrocytes expression clearly indicates reactive astrogliosis, which happens as a result of a feedback mechanism of the neurons at injured site as a healing process while in extreme cases leads to formation of scar. This finding is in line with the work of Bruccoleri and his colleagues that acute administration of trimethyltin which causes cell death, and the process of necrosis is accompanied by a microglia and astrocyte response in the hippocampus of adult male Wistar rats (Bruccoleri et al., 1998). Fiedorowicz and colleagues also reported a similar scenario, when they stated that the neurodegeneration caused by trimethyltin was associated with reactive astrogliosis in whole hippocampus of adult mice (Fiedorowicz et al., 2001).

Finally, quantitative analysis test was done to count p53 positive cells in the hippocampus of the rats and compare the outcomes between the rats that received normal saline and the rats that took single dose of trimethyltin. There was significant increase in number of p53 positive cells in the hippocampus of the rats in groups that received trimethyltin when compared to the numbers of p53 positive cells in the hippocampus of rats that were given normal saline. This implies that, the deleterious effect of trimethyltin was higher led to a reduced half-life of this gene, causing it to accumulate in the hippocampus either to initiate apoptosis or to stop the process of cell cycle to give time for cell repair. The finding of this study suggested that, trimethyltin was able to cause distinctive pro-apoptotic cell proliferation in the regions of the hippocampus of adult male Wistar rats. This is evidence that trimethyltin can initiate apoptosis in the hippocampus and this is in line with the works of Fiedorowicz and his colleagues, that suggested trimethyltin can initiated apoptosis in maurine hippocampal neurons of the dentate gyrus (Fiedorowicz et al., 2006), the work of Zhang 
and co-workers said that trimethyltin-induced apoptosis is associated with upregulation of inducible nitric oxide synthase and Bax in a hippocampal cell line (Zhang et al., 2006) and that apoptosis induced by trimethyltin in human neuroblastoma cells SY5Y is regulated by a balance and cross-talk by some growth factors signalling pathways (Qing et al., 2013).

The degenerative changes observed in this study as a result of exposure to trimethyltin, underlie the ability of trimethyltin to initiate cellular apoptosis and cause damage to the cells of the hippocampus of rats. This is in line with the studies of Donald et al., 1987; Thompson et al.,
1996; Geloso et al., 1997; Fiedorowicz et al., 2001, which suggested extensive damage to the neurons of the hippocampus of rats after exposure to trimethyltin.

This study was able to demonstrate trimethyltin induced neuronal injury by causing the activation of p53 proteins, increased astrogliosis and hyperphosphorylation of tau proteins within and around the cells of hippocampus in adult male Wistar rats. This implies that trimethyltin injury in adult rats, can serve as good model to study neurogenerative diseases.

CONFLICT OF INTEREST: None declared.

ACKNOWLEDGEMENTS: The immunohistochemical analysis of this work was done in the Histopathology Laboratory, National Hospital Abuja. This work was technically assisted by Mr Jonathan a Senior Laboratory Technician, National Hospital Abuja Nigeria. The histolochemical analysis of this work, was done in the Histopathology Laboratory, University Teaching Hospital Ilorin, with the technical assistance of Dr. Ibrahim Olatunde, a Consultant Histopathologist.

\section{REFERENCES}

1. Ajao MS, Olaleye O, Ihunwo AO. 2010. Melatonin potentiates cells proliferation in dentate gyrus following ischemic brain injury in adult rats. J Anim Vet Adv 9 (11): 1633-1638.

2. Bredesen DE, Rao RV, Mehlen P. 2006. "Cell death in the nervous system". Nat. 443 (7113): 796802.

3. Bruccoleri A, Brown H, Harry GJ. 1998. Cellular Localization and Temporal Elevation of Tumor Necrosis Factor-a, Interleukin-la, and Transforming Growth Factor-, 81 mRNA in Hippocampal Injury Response Induced by Trimethyltin. J Neurochem 71: (4) 1577-1586.

4. Colpo GD, Stimming EF, Rocha NP, Teixeira AL. 2017. Promises and pitfalls of immune-based strategies for Huntington's disease. Neural Regen Res 12(9) DOI: 10.4103/1673-5374.215245

5. Constantinidis, J. 1991. The hypotheses of zinc deficiency in the pathogenesis of neurofibrillary tangles. J Med Hypo 35(4):319-323.

6. Donald GR, Robert HG, Felix A. 1987. Quantitative Assessment of Trimethyltin Induced Pathology of the Hippocampus. Toxicol Pathol 15: 1, Printed in U.S.A. ISSN: 09 12-6233

7. Fiedorowicz A, Figiel I, Kaminska B, Zaremba M, Wilk S, Oderfeld-Nowak B. 2001. Dentate granule neuron apoptosis and glia activation in murine hippocampus induced by trimethyltin exposure. $\mathrm{Br}$ Res 912, 116

8. Fiedorowicz A, Figiel I, Zaremba M, Schliebs R, Oderfeld-Nowak B. 2006. IL-1 system in apoptosis of murine hippocampal neurons of dentate gyrus induced by trimethyltin administration. J Neurochem 85 (18): 1471-4159. 
9. Geloso MC, Corvino V, Cavallo V, Toesca A, Guadagni E, Passalacqua R, Michetti F. 2004. Expression of astrocyticnestin in the rat hippocampus during trimethyltin induced neuro degeneration. Neurosci 357: 103-106.

10. Geloso MC, Vinesi P, Michetti F. 1997. Calretinin-containing neurons in trimethyltin-induced neurodegeneration in the rat hippocampus: an immunocytochemical study. Exp Neurol 146, 6773.

11. Nilsberth C, Kostyszyn B, Luthman J. 2002. Changesin APP; PS1 and other factors related to Alzheimer's disease pathophysiology after trimethyltin-induced brain lesion in the rat. Neurotoxicol 4:625-636.

12. Ogundele, OM, Balogun WG, Cobham EA, Amin A, Ishola O A. 2015. Endothelial proliferation modulates neuron-glia survival and differentiation in ischemic stress. Ann Neurosci 22(3):150161

13. Price DL, Sisodia SS, Borchelt DR. 1998. Genetic Neurodegenerative Diseases: The Human illness and Transgenic Models. Sci 282 (5391), 1079-1083 DOI: 10.1126/science.282.5391.1079

14. Qing, Y, Liang Y, Du Q, Fan P, Xu H, Xu Y, Shi N. 2013. Apoptosis induced by Trimethyltin chloride in human neuroblastoma cells SY5Y is regulated by a balance and cross-talk between NF-kB and MAPKs signalling pathways. Mol Toxicol 87 (7): 1y273-1285.

15. Rubinsztein DC. 2006. The roles of intracellular protein-degradation pathways in neurodegeneration. Nat 443 (7113): 780-6.

16. Thompson TA, Lewis JM, Dejneka NS, Severs WB, Polavarapu R, Billingsley ML. 1996. Induction of apoptosis by organotin compounds in vitro: neuronal protection with antisense oligonucleotides directed against stannin. J Pharm Exp Therm 276, 1201-1216.

17. Woodruff ML, Baisden RH, Cannon RL, Kalbfleisch J, Freeman JN. 1994. Effects of trimethyltin on acquisition and reversal of a light-dark discrimination by rats. Physiol Behav 55: 1055-1061.

18. Yokel RA. 2000. Toxicology of Aluminium, Neurotoxicol 21(5)813-825.

19. Zhang L, Li L, Prabhakaran K, Borowitz JL, Isom GE. 2006. Trimethyltininduced apoptosis is associated with upregulation of inducible nitric oxide synthase and Bax in a hippocampal cell line. Toxicol App Pharm 216 (1): 34-43. 